\title{
Impurity-Induced Grain Boundary Strengthening in Polycrystalline Graphene
}

\author{
Fanchao Meng ${ }^{1}$, Dianyin $\mathrm{Hu}^{2,3}$, and Jun Song ${ }^{1 *}$
}

1. Department of Mining and Materials Engineering, McGill University, Montréal, Québec H3A 0C5, Canada

2. School of Energy and Power Engineering, Beihang University, Beijing 100191, China

3. Collaborative Innovation Center of Advanced Aero-Engine, Beijing 100191, China

\section{SUPPORTING INFORMATION}

\section{S1. Construction of symmetric tilt grain boundaries in graphene}

The construction of STGBs was illustrated in Figure S1. Starting from a perfect graphene sheet, a "V" notch (delimited by the two vectors in solid lines in Figure S1a) is then cut out. The two sides (i.e., Grain A and Grain B in Figure S1a) are then rotated to stitch with each other to form the GB (Figures S1b-d). ${ }^{1}$ The angle of the "V" notch is equal to the GB angle that can be prescribed by the vectors based on the two base vectors $\left(\boldsymbol{a}_{1}\right.$ and $\left.\boldsymbol{a}_{2}\right)$ of the graphene lattice. For $\theta$ $=16.4^{\circ}$, the vector is $(8,3)$, while the vectors are $(7,3),(5,2)$, and $(6,2)$ for STGBs with angles $\theta=9.5^{\circ}, \theta=13.2^{\circ}$, and $\theta=21.8^{\circ}$, respectively. Note that during the stitching process, some atoms in the overlap region need to be deleted to eliminate any discrepancy/artifacts along the GB line.

\footnotetext{
* Author to whom correspondence should be addressed. Email: jun.song2@mcgill.ca Tel.: +1 (514) 398-4592 Fax: +1 (514) 398-4492
} 


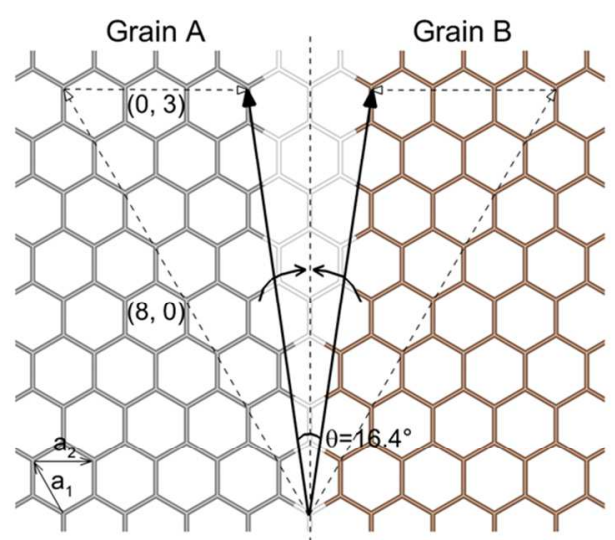

(a)

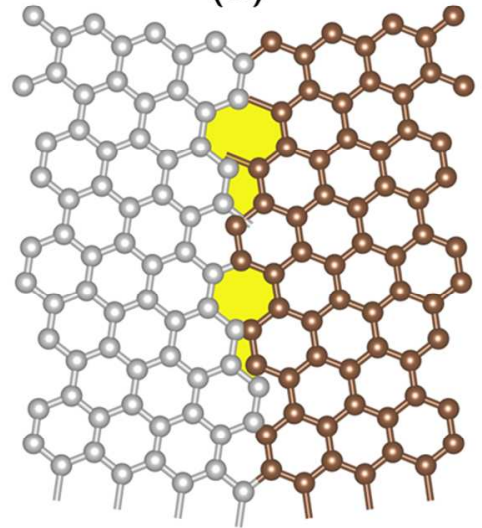

(c)

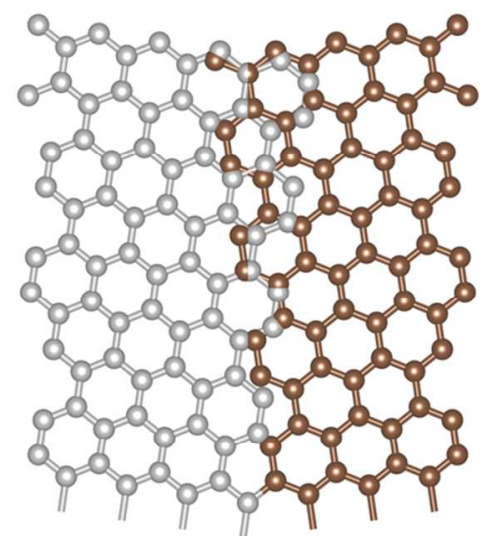

(b)

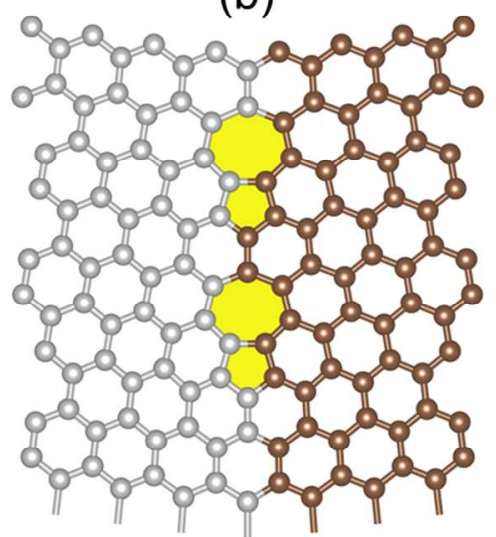

(d)

Figure S1. Illustration of the detailed process to construct a graphene STGB, including the steps of (a) defining the GB angle by two base vectors, (b) rotation of the two crystals, (c) removal of overlapped atoms and stitching, and (d) relaxation to attain the final equilibrium GB structures.

\section{S2. Elastic interactions of GBs and effects on fracture}

With GBs essentially composed of dislocations that of are a $1 / r$ field, ${ }^{2-3}$ elastic interactions between GBs will necessarily be present. We have performed calculations to examine the possible size dependence of our results. GB supercells with varying supercell width, i.e., $d_{x}$, were built for calculations, as illustrated in Figure S2. The stretching energy $\left(F_{s}\right)$ of the critical bond at different applied strains, and the fracture strain $\left(\varepsilon_{c}\right)$ with and without substitutional dopants, were examined as functions of $d_{x}$. Figure $\mathrm{S} 3$ shows the results for the $\Sigma 19$ $\left(\theta=13.2^{\circ}\right) \mathrm{STGB}$ as a representative system, where the values of $F_{s}$ of the critical bond at zero strain and a large strain of $6.5 \%$ as $d_{x}$ varies are plotted in Figure S3a, while the values of $\varepsilon_{c}$ for 
a pristine $\Sigma 19$ GB and a $\Sigma 19$ GB with a substitutional B atom doped at site $\alpha$ (see Figure 1 of the paper) as $d_{x}$ varies are plotted in Figure S3b. We see that both $F_{s}$ and $\varepsilon_{c}$ converge when $d_{x}$ is larger than $\sim 32 \AA$, regardless of the strain applied (for the evaluation of $F_{s}$ ) and the doping (for the evaluation of $\varepsilon_{c}$ ). In our study, the width used was ensured to be $>40 \AA$ to avoid any size dependence induced by mutual elastic interactions between GBs.

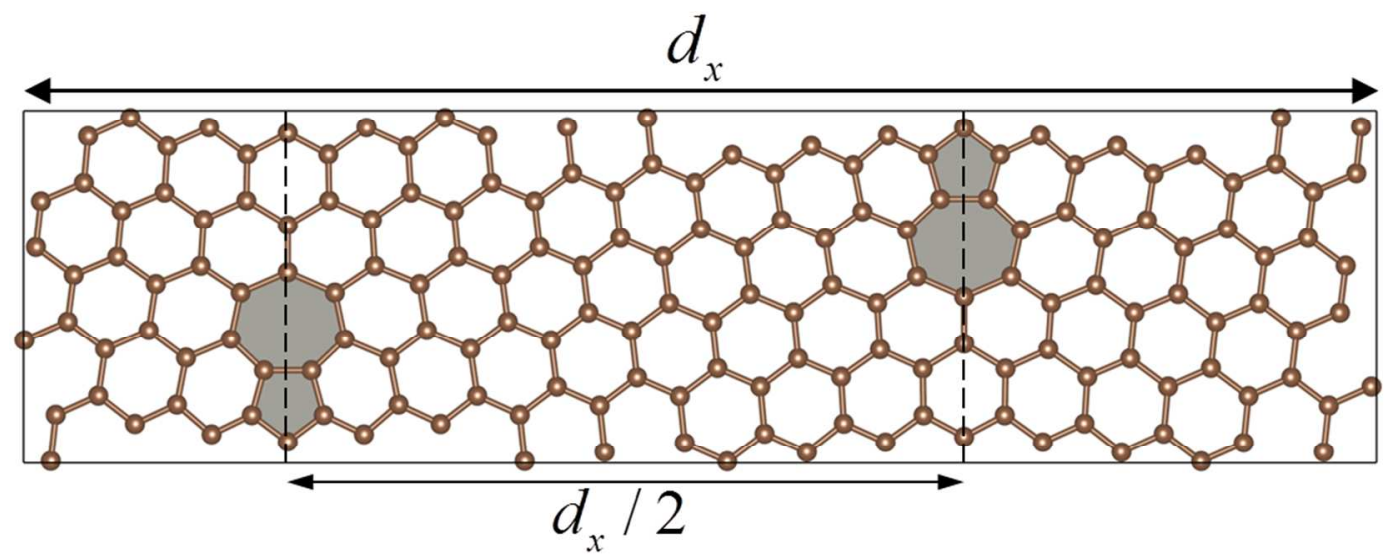

Figure S2. Illustration of a GB supercell (using the $\Sigma 19\left(\theta=13.2^{\circ}\right)$ STGB as a representative) of width $d_{x}$, where the two neighboring GBs is separated by a distance of $d_{x} / 2$. The dashed lines indicate the GB lines.
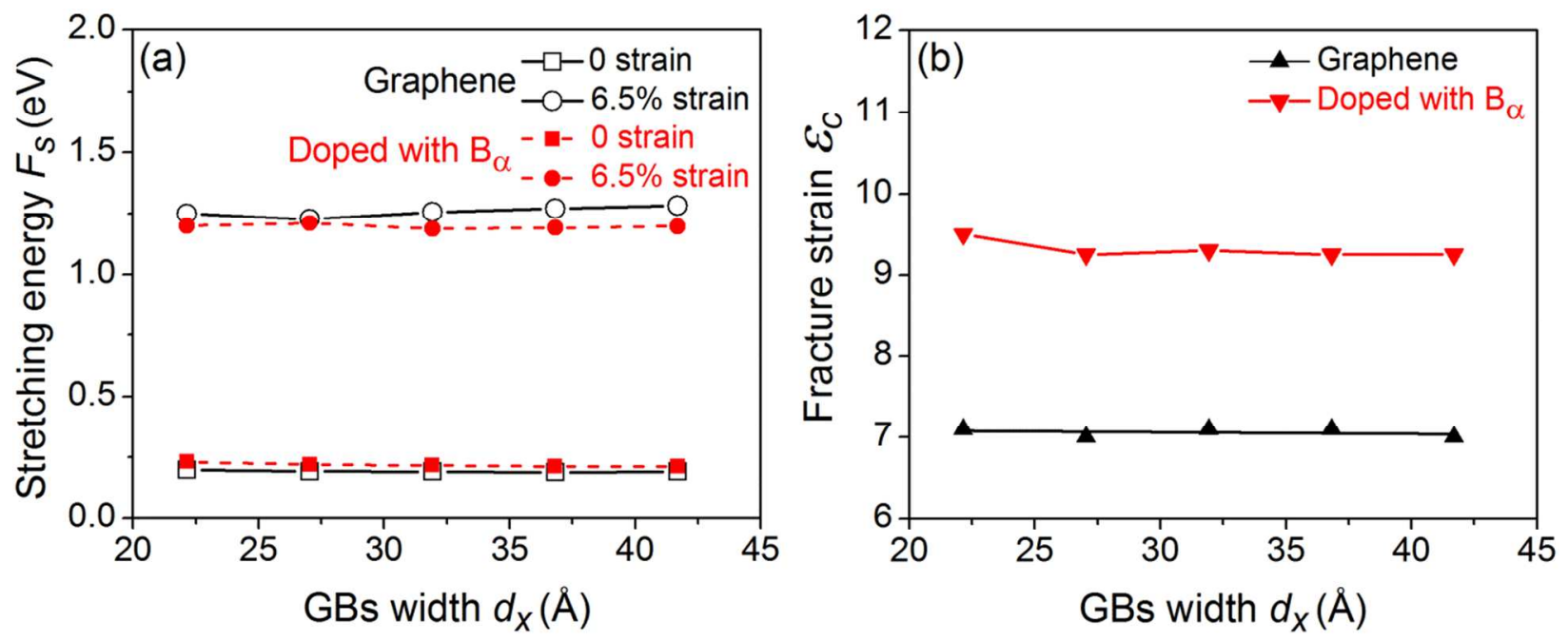

Figure S3. (a) Stretching energies at applied strains of 0 and $6.5 \%$ and (b) critical fracture strains for a pristine graphene GB and graphene GB with one B substitutional dopant at site $\alpha$, as functions of the GB width $d_{x}$. Here the $\Sigma 19\left(\theta=13.2^{\circ}\right)$ STGB is used as the representative system. 


\section{S3. Energetics of $\mathbf{B} / \mathbf{N}$ adatoms and effects on fracture}

Adatoms are an important class of dopants in graphene. To this end, we conducted a preliminary investigation of the adsorption of adatoms at GBs. Below we present our results, using the adsorption of $\mathrm{B} / \mathrm{N}$ adatoms at the $\Sigma 19 \operatorname{STGB}\left(\theta=13.2^{\circ}\right)$ as a representative case (see Figure S4 below). Defining the adsorption energy $\Omega_{a d s}$ of an adatom as (in a similar way as the formation energy of a substitutional atom $)^{4}$

$$
\Omega_{a d s}=E_{a d s}-E_{0}-\mu
$$

where $E_{0}$ is the total energy of the pristine graphene supercell, $E_{a d s}$ is the total energy of the supercell after the introduction of an adatom ( $\mathrm{B}$ or $\mathrm{N}$ ), and $\mu$ is the chemical potential for the adatom, which can be obtained from $\alpha$-boron bulk and $\mathrm{N}_{2}$ molecule for $\mathrm{B}$ and $\mathrm{N}$, respectively. The adsorption energies for some sample cases considered here are listed in Table S1, where we can see that the adsorption of $\mathrm{B}$ or $\mathrm{N}$ adatom at the $\mathrm{GB}$ is endothermic, in sharp contrast to the substitution of $\mathrm{B}$ or $\mathrm{N}$ which is exothermic (see Table 1 in the paper). Therefore, the adsorption of $\mathrm{B}$ or $\mathrm{N}$ adatoms is clearly not energetically preferable.

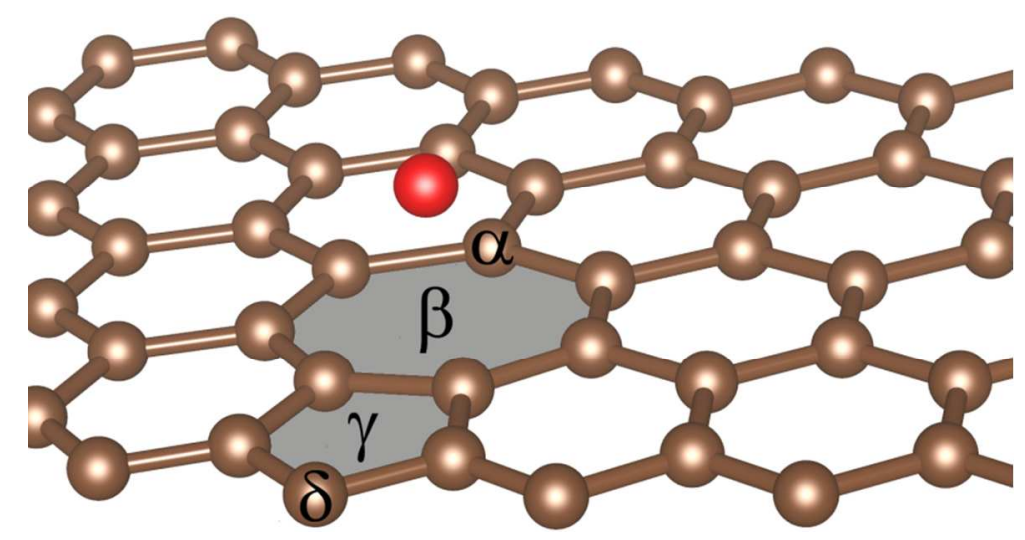

Figure S4. Illustration of possible adsorption positions for an adatom (B or N, colored red) at the representative $\Sigma 19\left(\theta=13.2^{\circ}\right)$ STGB. These sites are denoted as $\alpha, \beta, \delta$, and $\gamma$, representing heptagon tip, heptagon center, pentagon tip, and pentagon center, respectively. 
Table S1. Calculated adsorption energies for B or N adatom above the lattice sites considered in Figure S4

\begin{tabular}{ccccc}
\hline \hline \multirow{2}{*}{ Adatom } & \multicolumn{4}{c}{ Adsorption Energy (eV) } \\
\cline { 2 - 5 } & Above site $\alpha$ & Above site $\beta$ & Above site $\gamma$ & Above site $\delta$ \\
\hline $\mathrm{B}$ & 7.17 & 2.09 & 4.19 & 3.92 \\
$\mathrm{~N}$ & 3.43 & 3.44 & 4.58 & 3.21 \\
\hline \hline
\end{tabular}

Besides the energetics, we also performed some calculations to evaluate possible influence of adatoms on the fracture behaviors of graphene GBs. Generally we found that the introduction of adatoms at GBs does not alter the chemical nature of the critical bond ${ }^{\dagger}$. The adatoms however can lead to sizable out-of-plane deformation/buckling at the GB (see Figure S5 for an illustrative case of an $\mathrm{N}$ adatom adsorbed on top of site $\delta$ ), which helps release the elastic strain energy from the 5-7 pair. Nonetheless, according to Wei et. al., ${ }^{1}$ the amplitude of the local buckling and its subsequent influence on fracture diminish quickly under tensile straining, and thus the initial buckling will not affect the final fracture strength of polycrystalline graphene. Therefore, here the buckling induced by $\mathrm{B} / \mathrm{N}$ adatom is not expected to affect the GB fracture strength studied in our paper.

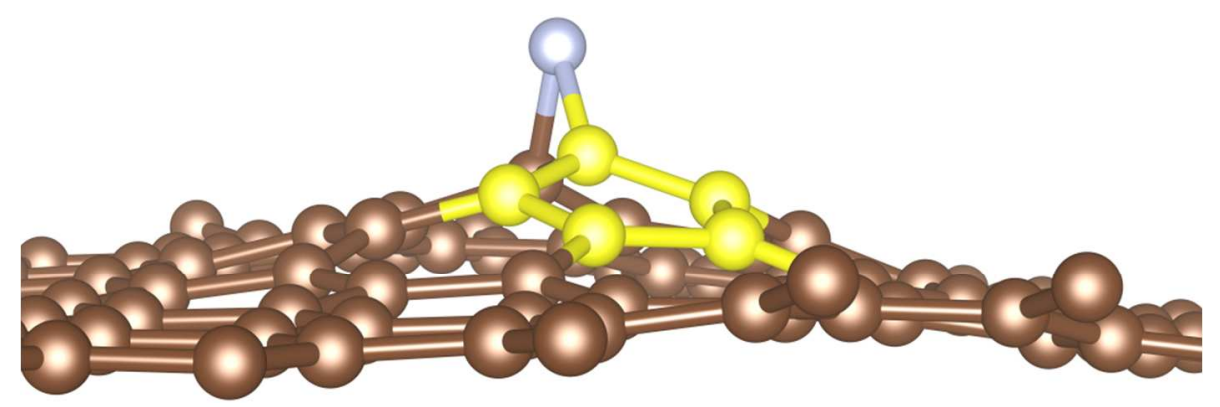

Figure S5. Out-of-plane deformation induced by $\mathrm{N}$ adatom (shown in light blue) initially put above $\delta$ site (see Figure S4). Atoms colored in yellow show the pentagon in the GB line.

\footnotetext{
${ }^{\dagger}$ It is worth noting that in certain special cases (where heavy local distortion exists) where the B adatom can get incorporated into the graphene lattice, in which case the B adatom essentially becomes a substitutional atom. Consequently the effects on fracture are the same as what was discussed in our paper.
} 


\section{S4. Critical bond in $B_{\varphi}$-doped graphene STGBs}

Under $B_{\varphi}$ doping, the critical bond remains the one shared by heptagon and hexagon, but becomes C-B in nature (see Figure S6a). Though the negative formation energy of B indicates that $\mathrm{C}-\mathrm{B}$ is chemically stronger than $\mathrm{C}-\mathrm{C}$ bond, we note that $B_{\varphi}$ doping also greatly affects the stretching energy of the $\mathrm{C}-\mathrm{C}$ bond immediately neighboring the critical bond. As shown in Figure S6b, we see that the stretching energy of the C-C bond is substantially lower than that of the critical C-B bond. This explains why the $\mathrm{C}-\mathrm{C}$ bond (despite chemically being weaker) becomes more resistant to the C-B bond.
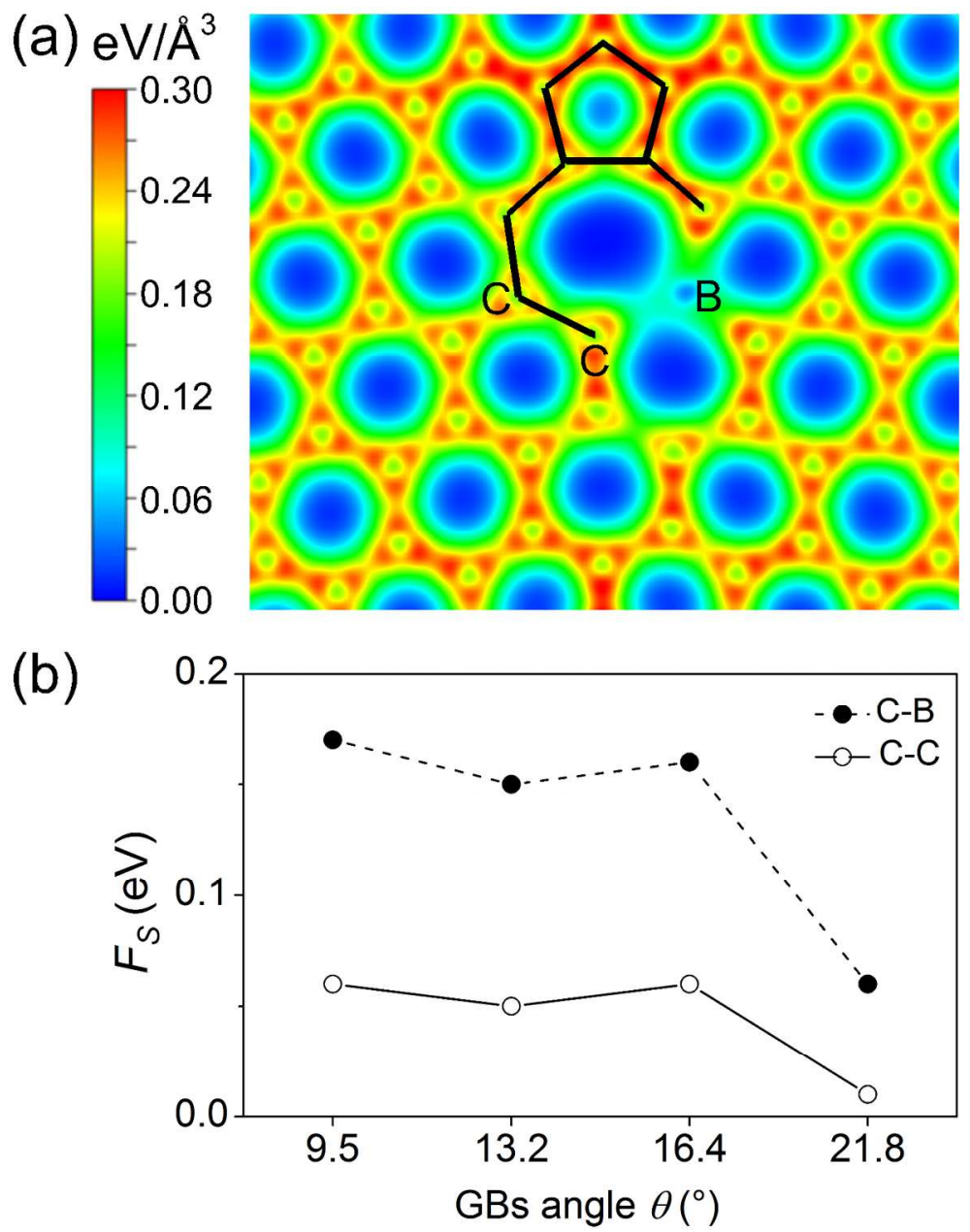

Figure S6. (a) The critical bond becomes C-B under $B_{\varphi}$ doping, where the $\Sigma 19\left(\theta=13.2^{\circ}\right)$ STGB is used as a representative. (b) Comparison of stretching energy of the critical C-B bond and its neighboring C-C bond for different graphene STGBs. 


\section{S5. Crack initiation under $B B$ doping for $\Sigma 7\left(\theta=21.8^{\circ}\right)$ STGB}

As mentioned in the main text, for the $\Sigma 7\left(\theta=21.8^{\circ}\right) \mathrm{STGB}$, the critical bond under $B B$ doping is the C-C bond shared by heptagon and pentagon (see Figure S7a), different from the other STGBs. One possible reason is that the stretching energy of the $\mathrm{C}-\mathrm{C}$ bond in $\Sigma 7\left(\theta=21.8^{\circ}\right)$ STGB becomes higher than that of C-B bond (see Figure S7b).
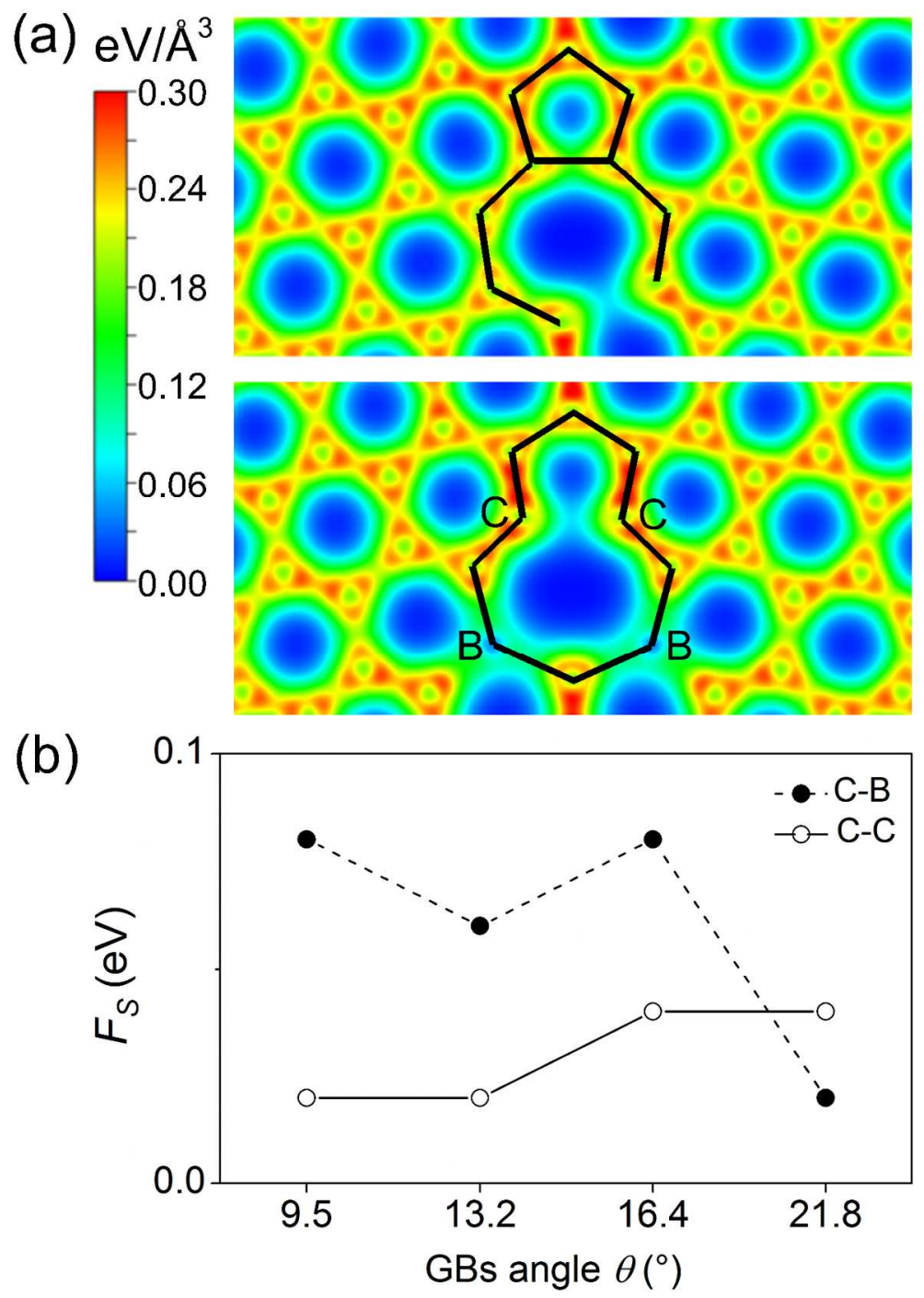

Figure S7. (a) Crack initiation for the $\Sigma 7\left(\theta=21.8^{\circ}\right)$ STGB (top) in absence of dopants and (bottom) under $B B$ doping, showing different crack initiation locations while the bond remains $\mathrm{C}-\mathrm{C}$ in nature. (b) Comparison of stretching energy of the $\mathrm{C}-\mathrm{B}$ bond (shared by heptagon and hexagon) the critical C-C bond (shared by heptagon and pentagon) for different graphene STGBs. 


\section{S6. Crack deflection under $N N$ doping for $\Sigma 49$}

As noted from Figure $5 \mathrm{~b}$, we see that the trends of $\alpha_{\Gamma}$ and $\Delta \varepsilon_{p}$ show some notable deviation for the $\Sigma 49\left(\theta=16.4^{\circ}\right)$ STGB. This is likely due to the fact that the two $\mathrm{N}$ dopants reside at $\lambda^{*} \lambda^{*}$ along $\Sigma 49$, which is $\sim 10.5 \AA$ away from the location where fracture initiates (while for other STGBs the two $\mathrm{N}$ dopants sit at $\lambda \lambda$, being in the immediate vicinity of the fracture initiation site) as shown in Figure S8. For $\Sigma 49$, the $N N$ doped pentagon is found to add some additional resistance to the first stage of propagation before crack deflection occurs, which contributes to increasing $\Delta \varepsilon_{p}$.

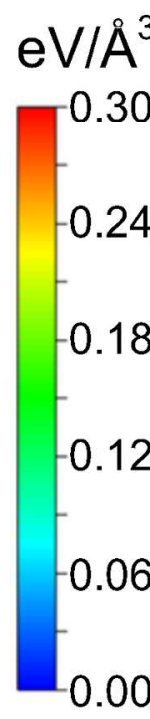

(a)

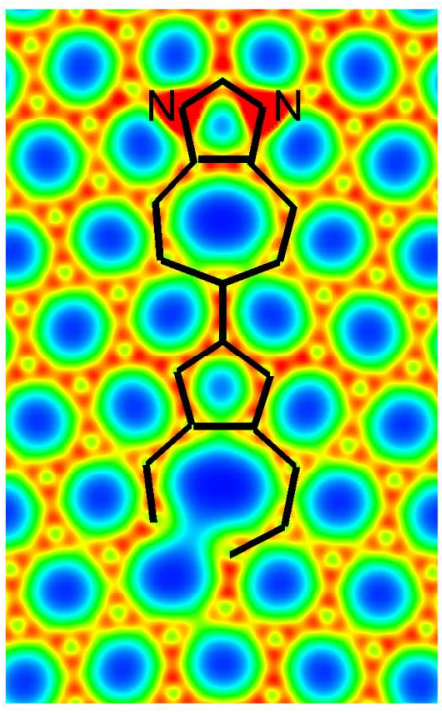

(b)

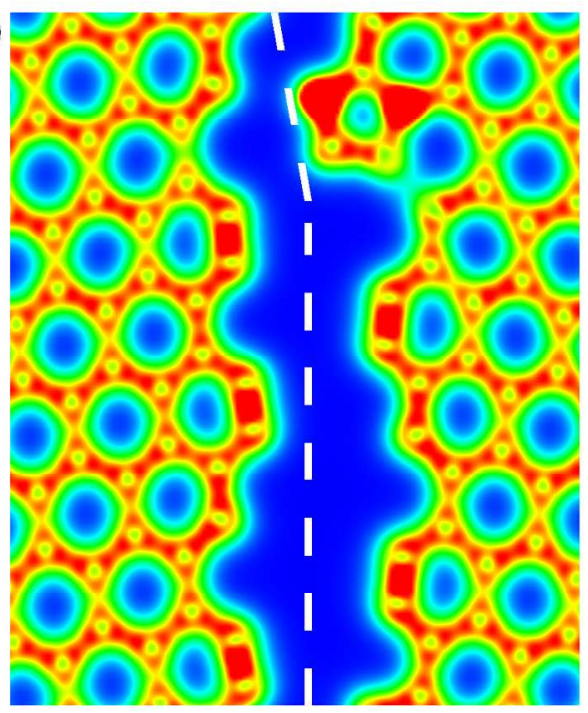

Figure S8. Fracture initiation event along the $\Sigma 49\left(\theta=16.4^{\circ}\right)$ STGB, where the breakage at the critical bond (a) and subsequent propagation process (b) are illustrated by the charge density plots, which show that an initial propagation is needed before deflection can occur.

\section{S7. Illustration of crack propagation under $B \boldsymbol{B}$ and $B^{2} N^{2}$ doping}

As stated in S5, expect the GB with $\theta=21.8^{\circ}$, GBs doped with $B B$ and $B^{2} N^{2}$ will initiate from the C-B bond, being the critical bond shared by the heptagon and hexagon, and will fracture along GBs with or without crack deflection. Figure S9 shows the fracture initiation and propagation processes using $\Sigma 19\left(\theta=13.2^{\circ}\right) \mathrm{STGB}$ and $\Sigma 37\left(\theta=9.5^{\circ}\right)$ as representatives. Under 
$B^{2} N^{2}$ doping, it is found that crack deflection happens along low strength STGBs (i.e., $\Sigma 37$ and $\Sigma 49$ ) but not at high strength STGBs (i.e., $\Sigma 19$ and $\Sigma 7$ ). The higher strength of $\Sigma 19$ and $\Sigma 7$ STGBs possibly originates from the mutual cancellation of the stress fields of 5 and 7 defects, in which cases $B B$ doping can weaken the effects of $N N$ doping more than that of low strength $\Sigma 37$ and $\Sigma 49$ STGBs.

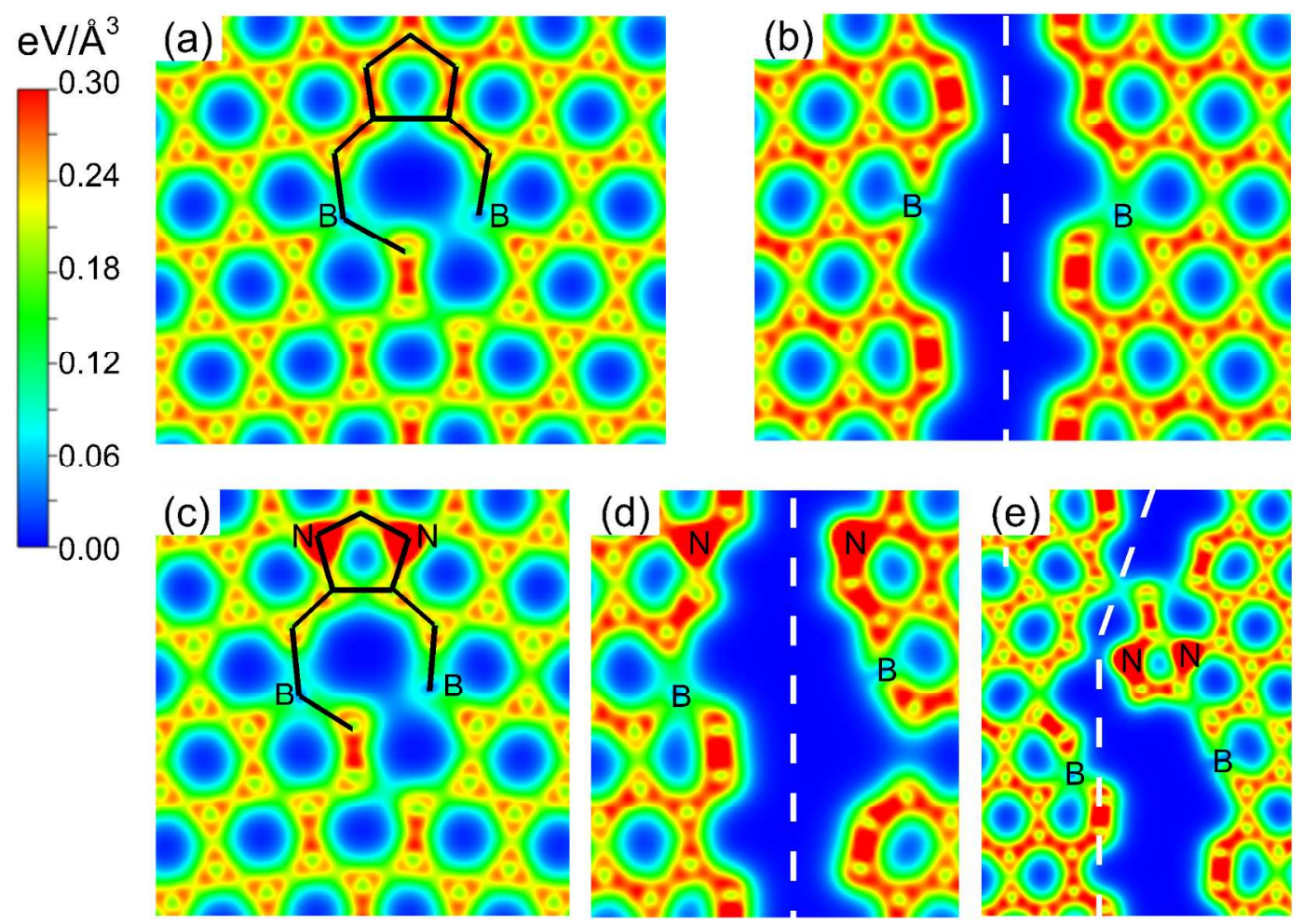

Figure S9. (a) Typical fracture initiation event represented by the $\Sigma 19\left(\theta=13.2^{\circ}\right)$ STGB under $B B$ doping, where the critical bond is $\mathrm{C}-\mathrm{B}$ (except GB with $\theta=21.8^{\circ}$, see S3 for details) and (b) subsequent propagation through GBs. (c) Typical fracture initiation event represented by the $\Sigma 19\left(\theta=13.2^{\circ}\right)$ STGB under $B^{2} N^{2}$ doping, where the critical bond is C-B and subsequent propagation process in (d) through GBs represented by $\Sigma 19\left(\theta=13.2^{\circ}\right)$ STGB and (e) deflected from GBs represented by $\Sigma 37\left(\theta=9.5^{\circ}\right)$ STGB, respectively. 


\section{SUPPLEMENTARY REFERENCE}

(1) Wei, Y.; Wu, J.; Yin, H.; Shi, X.; Yang, R.; Dresselhaus, M. The Nature of Strength Enhancement and Weakening by Pentagon-Heptagon Defects in Graphene. Nat. Mater. 2012, 11, 759-763.

(2) Meng, F.; Chen, C.; Song, J. Dislocation Shielding of a Nanocrack in Graphene: Atomistic Simulations and Continuum Modeling. J. Phys. Chem. Lett. 2015, 6, 4038-4042.

(3) Song, Z.; Xu, Z. Topological Defects in Two-Dimensional Crystals: The Stress Buildup and Accumulation. J. Appl. Mech. 2014, 81 (091004), 1-5.

(4) Mavrikakis, M.; Hammer, B.; Nørskov, J. K. Effect of Strain on the Reactivity of Metal Surfaces. Phys. Rev. Lett. 1998, 81 (2819), 1-4. 